\begin{tabular}{c} 
International Journal of Engineering \& Technology, $7(2.23)(2018) 4-8$ \\
International Journal of Engineering \& Technology \\
SPC \\
Website: www.sciencepubco.com/index.php/IJET \\
Research paper \\
\hline
\end{tabular}

\title{
Prediction the wear of sliding bearings
}

\author{
Dykha Aleksandr ${ }^{1 *}$, Marchenko Dmitry ${ }^{2}$ \\ ${ }^{1}$ Khmelnitskyi National University, Ukraine \\ ${ }^{2}$ Mykolaiv National Agrarian University, Ukraine \\ *E-mail:tribosenator@gmail.com
}

\begin{abstract}
The problem of developing a calculation-experimental method for calculating wear of a sliding bearing based on a two-factor wear model (contact pressure - sliding velocity) with identification of wear resistance parameters was considered. On the basis of the proposed wear model, the wear-contact problem for a cylindrical sliding bearing was solved. The equation of equilibrium for medium pressures and the approximating function of linear wear from the arc of contact between the shaft and the bushing were used as the determining equations. To identify parameters of wear resistance in the wear model, a calculation-experimental method for determining calculated dependences of wear resistance parameters was developed on the basis of the wear test by the «cone - three balls» scheme. The results of wear tests of bronze conical specimens with a variable wear spot and two values of sliding velocity were taken as a base. The obtained results were recommended for predicting wear of sliding bearings at the design stage and optimizing their design and operational parameters.
\end{abstract}

Keywords: sliding bearing, wear-contact problem, wear model, wear testing, wear resistance parameters.

\section{Introduction}

The current stage in development of tribology is characterized by creation of methods for calculating the wear of friction units. It was believed for a long time that creation of such methods is impossible because of an extreme complexity of wear processes. At the same time, if there are no methods of calculation at the stage of designing and machine creation, then respectively there will be no methods for predicting wear resistance and durability of the friction units. Development of analytical methods for calculating wear resistance of tribosystems is complicated by nonlinearity of wear models because of complex interrelationships of mechanical, thermophysical and frictional properties. To obtain correct results, analytical methods for calculating tribosystems require a number of parameters that characterize real operating conditions. Cylindrical sliding bearings in many manufacturing, transport and power machines are among the main components determining durability and reliability of the machine as a whole. Thus, creation of calculation and experimental methods and models of wear resistance for predicting wear-related durability of cylindrical sliding bearings taking into account the variety of operating conditions is a focal scientific problem.

\section{Literature review and problem statement}

Creation of methods for calculation and wear test of cylindrical sliding bearings is currently being given considerable attention. For example, on the basis of a cumulative wear model, solution of a tribocontact problem for a sliding bearing was performed in work [1]. In this case, the shaft shape was not cylindrical but had slight facets of various kinds. The proposed solution was rather complicated for practical implementation since it required breaking of the wear zone into discrete parts and did not take into ac- count technological features of making shafts with a specified faceting. In work [2], analysis of variation of the contact pressure during wear of the coating in a thrust sliding bearing was carried out at a nonlinear form of the wear law. The proposed algorithm for solving the wear-contact problem for the thrust bearing will have material complications in the case of cylindrical bearing. Solution of an inverse wear-contact problem for identifying parameters of dependence of the wear rate on pressure and sliding velocity with accounting for their distribution over the contact spot was made in [3]. On the basis of experimental study of wear by the "finger-disk" scheme, expressions were derived for determining these parameters. However, the assumption of permanence of the wear spot accepted in this work in accordance with the test pattern did not let use of the obtained solution for the test schemes with a variable contact (wear) spot of a "ball - ball" or "cone ball" type. A calculation-experimental method for determining tangential contact frictional stresses using a variational principle was proposed in work [4]. In this case, a quadratic functional of deviation of the experimental function of tangential stresses from the equilibrium condition was constructed. The authors of paper [5] have carried out a theoretical analysis of kinetics of wear of spherical specimens in the process of testing on a four-ball friction machine. However, the described kinetic model has a phenomenological character and is based on the principles of behavior of open thermodynamic systems. The authors of [6] proposed theoretical dependences for a wear testing method using a standard four-ball scheme with determination of wear resistance parameters The approximating function of the wear spot diameter on the friction path obtained by the results of wear tests was taken as the base of the method. The general methodology of this study can be used to develop a theory of test methods for other geometric schemes. For numerical simulation of the wear process for a radial sliding bearing, the finite element method was used in [7]. In this case, the contact conditions were modeled by the Lagrange-Euler complex and evolution of local wear was modeled by the Archard equation. As a result of this simulation, dependences of the contact pressures on wear and the influence of the bearing clearance on 
these dependences were obtained. A calculation-experimental method for determining viscous friction characteristics for a cylindrical sliding support using a pendulum scheme of damped oscillations was proposed in [8]. The method for determining lubricant viscosity can be recommended to obtain viscosity values in wear calculations of sliding bearings.nIn work [9], dependence of the surface layer stresses resulting from the shaft-sleeve contact interaction in the sliding bearing on its wear resistance was considered. Instead of contact pressures, surface stressed state which was numerically estimated by the finite element method was taken as the determining factor of the wear rate. It is difficult to use this approach in the engineering practice of calculating bearing wear at the design phase.

This work's objective was to create a calculation-experimental method for calculating the wear of sliding bearings based on a two-factor wear model (contact pressure - sliding velocity) with identification of their wear resistance parameters.

\section{Solution of the wear-contact problem for a cylindrical sliding bearing}

The wear-contact problem is the problem of determining maximum linear wear in the bearing depending on the friction path (working life). In this case, the bearing parameters of design, loading and kinematics as well as regularity of wear depending on wear-resistance parameters were taken as the starting data.

By their design scheme, cylindrical sliding bearings represent an internal contact of two cylinders of close radii with a radial clearance $\Delta$ (Fig. 1). The shaft with radius $R_{I}$ is loaded with force $Q$ and rotates at a sliding velocity $V$ in the bearing bush 2 having radius $R$. In the process of force interaction of the shaft and the bearing bush, the contact pressure $\sigma$ distributed over the arc of contact $2 \varphi_{0}$ arises at the place of contact of two cylinders.

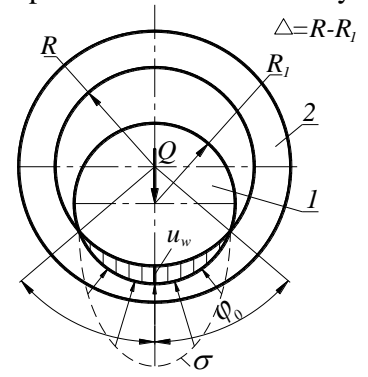

Fig. 1: Calculation scheme of a cylindrical sliding bearing

For analytical solution of the problem of the sliding bearing wear calculation, in addition to the indicated geometrical, force and kinematic parameters, a mathematical form of wear regularity is also taken as the initial value. Representation of such regularities in a form of dependence of the wear rate on the determining parameters of the process (contact pressure, sliding velocity, temperature, etc.) was of the greatest use. Here, we propose regularity (model) of wear in a form of a dimensionless set of determining factors:

$\frac{\mathrm{d} u_{W}}{\mathrm{~d} S}=C_{W}\left(\frac{f \sigma}{H B}\right)^{n}\left(\frac{V R}{v}\right)^{p}$,

where $u_{W}$ is bearing wear; $S$ is friction path; $f$ is coefficient of friction in the shaft-bushing pair; $\sigma$ is normal contact pressure; $H B$ is hardness of the bushing material; $V$ is sliding velocity; $R$ is bearing (bushing) radius; $v$ is kinematic oil viscosity; $C_{W}, n, p$ are parameters of wear resistance.

In the basic dependence (1), value of the contact pressure will be determined from the mean values (equation of equilibrium):

$\sigma=\frac{Q}{2 b R \varphi_{0}}$ where $Q$ is load acting on the bearing; $b$ is width of the contact between the shaft and the bushing; $\varphi_{0}$ is half-angle of the contact between the shaft and the bushing.

The relationship of the maximum wear $u_{W}$ and the contact angle is determined from geometry of the internal contact of two cylinders with an initial radial clearance $\Delta$ from the dependence:

$u_{W}\left(\varphi_{0}\right)=\Delta\left(\sec \varphi_{0}-1\right)$.

For further use, it is convenient to represent function $\sec \varphi_{0}-1$ in a form of a power approximation. For the range of changes in the contact angle $0 \ldots \pi / 2$, use the Excel program to construct graphical dependence of function $\sec \varphi_{0}-1$ on the contact angle $\varphi_{0}$ (Fig. 2) and obtain a power approximation of this function.

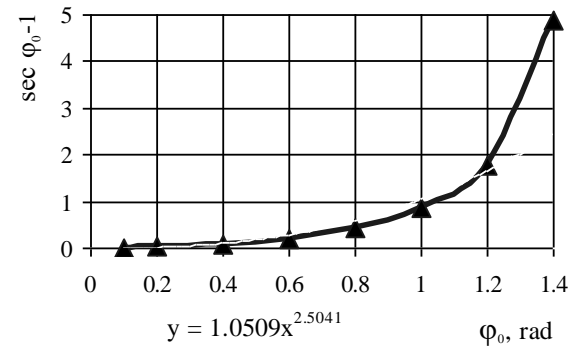

Fig. 2: Power approximation of function $\sec \varphi_{0}-1$ on the contact angle $\varphi_{0}$

With a sufficient accuracy, it can be assumed that $\sec \varphi_{0}-1 \approx \varphi_{0}^{2,5}$.

Thus, expression (3) can be represented in the following form:

$u_{w}=\Delta \varphi_{0}(s)^{2 \frac{1}{2}}$.

Differentiate the dependence (5) in the friction path $s$ :

$\frac{\mathrm{d} u_{w}}{\mathrm{~d} s}=2 \frac{1}{2} \Delta \varphi_{0}(s)^{1 \frac{1}{2}} \frac{\mathrm{d} \varphi_{0}}{\mathrm{~d} s}$.

Equate (1) and (6) and substitute into the left-hand side the expression for the contact pressure (2) to obtain:

$c_{W}\left(\frac{V R}{v}\right)^{p}\left(\frac{f}{H B}\right)^{n}\left(\frac{Q}{2 b R \varphi_{0}}\right)^{n}=2 \frac{1}{2} \Delta \varphi_{0}(s)^{1 \frac{1}{2}} \frac{d \varphi_{0}}{d s}$.

Transform (7) to the form:

$\frac{c_{W}}{2,5 \Delta}\left(\frac{V R}{v}\right)^{p}\left(\frac{f Q}{H B 2 b R}\right)^{n}=\varphi_{0}^{n+1,5} \frac{d \varphi_{0}}{d s}$.

or

$\frac{2 c_{W}}{5 \Delta}\left(\frac{V R}{v}\right)^{p}\left(\frac{f Q}{H B 2 b R}\right)^{n} d s=\varphi_{0}^{n+1,5} d \varphi_{0}$.

This is an ordinary differential equation with separating variables. Integrate equation (9) to obtain:

$\frac{\varphi_{0}^{n+2,5}}{n+2,5}=\frac{2 c_{W}}{5 \Delta}\left(\frac{V R}{v}\right)\left(\frac{f Q}{H B 2 b R}\right)^{n} s+C$.

Assuming that the contact angle $\varphi_{0}=0$ at the initial moment of the wear process $(s=0)$, the integration constant $C=0$.

Thus, the dependence of the contact angle on the friction path takes the form:

$\varphi_{0}=\left[\frac{(n+2,5) 2^{1-n} c_{W}}{5 \Delta}\left(\frac{V R}{v}\right)^{p}\left(\frac{f Q}{H B b R}\right)^{n} s\right]^{\frac{1}{n+2,5}}$.

Or finally, taking into account dependence (5), the calculation formula for dependence of the maximum wear $u \mathrm{w}$ in the sliding bearing on the magnitude of the friction path $s$ will be:

$u_{W}=\Delta\left[\frac{(n+2,5) 2^{1-n} c_{W}}{5 \Delta}\left(\frac{V R}{v}\right)^{p}\left(\frac{f Q}{H B b R}\right)^{n} s\right]^{\frac{2,5}{n+2,5}}$.

In the obtained formula (12), the wear resistance parameters $C_{W}, n$, $p$ show the degree of influence of the determining factors of the bearing wear process. As a rule, their numerical values are deter- 
mined experimentally by laboratory tests. On the one hand, these tests will not be cumbersome with the use of geometrically simple specimens and on the other hand, the test conditions tend to be as close as possible to the actual unit operating conditions in order to obtain a more adequate wear model. Further, the procedure for calculating wear resistance parameters from the results of laboratory wear tests is considered for a complete identification of formula (12).

\section{Identification of wear resistance parameters for the method of wear test}

Balls, cylinders, rollers, rectangular prisms, etc. were used in laboratory tests as specimens. Here, it was proposed to use the "cone - three balls" scheme of test to determine wear characteristics. Standard $12.7 \mathrm{~mm}$ diameter steel balls made of steel SH15/1.3505 were used as control specimens. A conical specimen of a corresponding bearing material was used as a test specimen. Conical specimens are easily prepared and are characterized by the wear spot dimensions changing during the test. The change of the wear spot leads to a change of contact pressures which makes it possible to obtain a set of test results by the load characteristics without changing the external load on the conical specimen. Below, a calculation and experimental procedure for identifying wear resistance parameters $C_{W}, n, p$ in the model of wear (1) for testing by the "cone - three balls" scheme is considered.

The calculation "cone - three balls" test scheme is shown in Fig. 3. Three balls 1, 2, 3 of the same radius $R$ are situated on the plane so that their centers form an equilateral triangle $\mathrm{O}_{1} \mathrm{O}_{2} \mathrm{O}_{3}$. The cone 4 with vertex angle $\gamma$ is contacting the lower placed balls at points $A_{1} A_{2} A_{3}$. Force $Q$ applied to the cone is transmitted to the lower balls along perpendiculars to the cone generatrix with equal forces $Q_{1}=Q_{2}=Q_{3}$
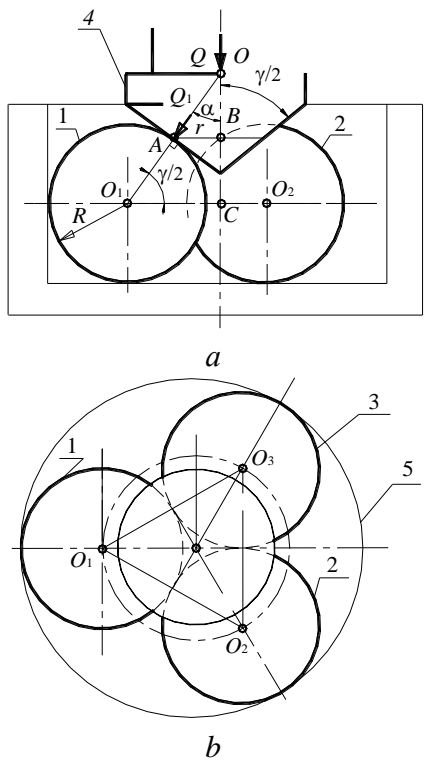

Fig. 3: The calculation "cone - three balls" test scheme: front view (a); top view $(b)$

The cage 5 ensures immobility of the balls under action of loading from the vertical force and a moment around the vertical axis. The forces acting on the balls are expressed through a common force by the relation:

$$
Q_{1}=Q_{2}=Q_{3}=\frac{Q}{3 \cos (\gamma / 2)} \text {. }
$$

To determine linear sliding velocity of the cone on the balls, it is necessary to know the distance $r$ from the cone rotation axis to the points of contact with the balls. From similarity of the triangles $O O_{l} C$ and $O A B$, the following is obtained:
$r=O_{1} C\left(1-\frac{R}{O O_{1}}\right)$

The value of $O_{l} C$ is defined as the radius of the circle circumscribed around the regular triangle $\mathrm{O}_{1} \mathrm{O}_{2} \mathrm{O}_{3}: \quad O_{1} \mathrm{C}=\frac{2 \sqrt{3}}{3} \mathrm{R}$.

Then from a right-angled triangle (Fig. 2):

$$
O O_{1}=\frac{O_{1} C}{\cos \gamma / 2}=\frac{2 \sqrt{3} R}{3 \cos \gamma / 2} \text {. }
$$

After intermediate substitutions, the following is obtained:

$$
r=R\left(\frac{2 \sqrt{3}}{3}-\cos (\gamma / 2)\right) \text {. }
$$

Take the shape of the worn-out cone surface as a circular groove with a profile radius $a$. Assume also that the contact pressure under the rigid wear-free ball is evenly distributed over the worn-out cone surface. Then the following relation holds:

$\sigma=\frac{Q_{1}}{\pi a^{2}}$.

The relation of the maximum wear $u_{W}$ with dimensions of the wear spot $a$ is determined from the geometry of contact of the conjugated cone of radius $R$ with a cylinder of radius $r$. With a sufficient accuracy, the sought dependence is represented as:

$u_{W}(S)=\frac{a(S)^{2}}{2 R^{*}}$,

where $: R^{*}=\frac{R r}{R+r}$.

In determining wear resistance parameters, use equivalent radius of the ball - cone contact in the model of wear resistance (1) instead of the bearing radius.

Represent the experimental dependence of the radius of a circular groove worn out in the cone as a power approximation:

$a(S)=c S^{\beta}$,

where $c$ and $\beta$ are the approximation parameters determined from the results of the wear tests.

Integrate the regularity of wear (1) to obtain an integral form of the model of cone wear:

$u_{W}(S)=C_{W} \int_{0}^{S}\left(\frac{f \sigma(S)}{H B}\right)^{n}\left(\frac{V R^{*}}{v}\right)^{p} \mathrm{~d} S$.

Substitute the expression for wear (17) in the left side of the obtained dependence and the expression for the contact pressure (16) in the right-hand side to obtain:

$\frac{a^{2}(S)}{2 R^{*}}=C_{W} \int_{0}^{S}\left[\left(\frac{f Q_{1}}{\pi \bar{a}^{2}(S)}\right) \frac{1}{H B}\right]^{n}\left(\frac{V R^{*}}{v}\right)^{p} \mathrm{~d} S$,

or taking into account (18) and integrating along the friction path, the following is obtained:

$\frac{c^{2} S^{2 \beta}}{2 R^{*}}=C_{W}\left(\frac{f Q_{1}}{c^{2} \pi H B}\right)^{n}\left(\frac{V R^{*}}{v}\right)^{p} \frac{S^{1-2 \beta n}}{1-2 \beta n}$.

From the condition of satisfiability of equation (21) for all values of $S$, it follows that:

$2 \beta=1-2 \beta n$,

whence:

$n=\frac{1-2 \beta}{2 \beta}$.

To determine parameter $p$, tests should be carried out at two values of the sliding velocity $V_{1}$ and $V_{2}$ from which two groups of experimental data with approximating functions are obtained:

$a_{1}=c_{1} S^{\beta} ; a_{2}=c_{2} S^{\beta}$.

Substitute expressions (24) into (21) and obtain a system of equations: 


$$
\left.\begin{array}{l}
\frac{c_{1}^{2} \beta}{R^{*}}=C_{W}\left(\frac{f Q_{1}}{c_{1}{ }^{2} \pi H B}\right)^{n}\left(\frac{V_{1} R^{*}}{v}\right)^{p} ; \\
\frac{c_{2}{ }^{2} \beta}{R^{*}}=C_{W}\left(\frac{f Q_{1}}{c_{2}{ }^{2} \pi H B}\right)^{n}\left(\frac{V_{2} R^{*}}{v}\right)^{p} \cdot
\end{array}\right\}
$$

Divide the first equation by the second and obtain the following after transformations:

$$
\left(c_{1} / c_{2}\right)^{2 n+2}=\left(V_{1} / V_{2}\right)^{p} \text {. }
$$

whence:

$$
p=(2 n+2) \frac{\lg \left(c_{1} / c_{2}\right)}{\lg \left(V_{1} / V_{2}\right)} \text {. }
$$

To determine coefficient $K_{W}$, use one of the equations

$$
C_{W}=\frac{\beta c_{1}^{2 n+2}}{R^{*}}\left(\frac{3 \pi H B \cos \alpha}{f Q}\right)^{n}\left(\frac{v}{V_{1} R^{*}}\right)^{p} .
$$

Thus, the calculation-experimental procedure for identifying wear resistance parameters for the "cone - three balls" scheme of wear test based on the two-factor wear model of the sliding bearing (contact pressure, sliding velocity) was proposed

\section{Experimental setup and the test procedure}

The experimental setup for the "cone - three balls" scheme of testing is shown in Fig. 4.

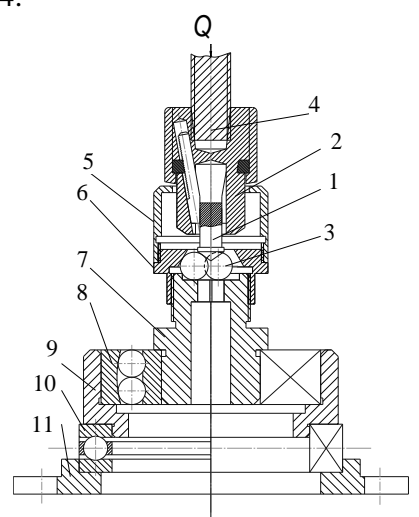

Fig. 4: The experimental setup

The test specimen 1 with a hardened conical surface is fixed in a universal self-centering drilling chuck 2 . The specimen 1 is pressed with a vertical load $Q$ onto the lower placed balls made of a ball bearing steel and is given a rotational motion from the spindle 4 of the experimental setup. The control ball specimens 3 are placed on the flat hardened surface of the support 7 and are centered by a special nut 6 with a conical working surface. Before testing, the cup 5 is filled with lubricant. A two-row self-centering bearing 10 located in the bottom housing 11 of the setup is used for self-adjustment of the specimens.

The tests were carried out under the following conditions. The vertical load acting on the conical specimen was taken equal to $Q=100 \mathrm{~N}$. The forces transmitted to the lower balls along the normal were determined from formula (2): $Q_{l}=40,6 \mathrm{~N}$. The cone angle of the conical sample was $\gamma=110^{\circ}$. Diameter of the lower control ball specimens was $\mathrm{D}=12.7 \mathrm{~mm}$. The radius of the circular slipping path of the conical sample over the balls was calculated by formula (4): $r=3,68 \mathrm{~mm}$. The equivalent radius of the ball cone contact will be $R^{*}=2.35 \mathrm{~mm}$. The tests were carried out at two rotation velocities of the setup spindle: $n_{1}=500 \mathrm{~min}^{-1}$ and $n_{2}=1000 \mathrm{~min}^{-1}$. These rotation velocities corresponded to linear sliding velocities for the conical sample: $V_{l}=192 \mathrm{~mm} / \mathrm{s} ; V_{2}=384$ $\mathrm{mm} / \mathrm{s}$. The conical samples were made of tin-phosphorous bronze CuSn10P having hardness $H B=90 \mathrm{MPa}$. The balls were made of ball-bearing steel SH15/1.3505. The samples were lubricated with engine oil Magnum 15W - 40 (TNK, Ukraine) having kinematic viscosity $v=40 \mathrm{~mm}^{2} / \mathrm{s}$ at the operating temperature. Friction coefficient of bronze rubbing against lubricated steel was taken $f=0.08$. The cone wear in the form of an annular groove on its generatrix was measured by the width of the worn-out groove using an МБС10 microscope with an accuracy of $0.05 \mathrm{~mm}$. A special device for locating the conical sample in the measurement plane was used in measurements. The depth of the worn-out groove was taken as the maximum normal wear of the bushing. The degree of wear can be calculated from the width of the worn-out groove according to geometric relationships if the shape of the groove profile is admitted to be circular. To determine shape of the worn surface and the amount of linear wear $u \mathrm{w}$, a projector with a $\times 77$ magnification on the screen was used.A nalysis of the worn out groove profile at a $\times 77$ magnification by the projector confirms its maximum closeness to a circular shape with a diameter equal to the ball diameter $D=12.7 \mathrm{~mm}$.

For example, a bronze specimen was tested under load $Q=100 \mathrm{~N}$ at sliding velocity $V=0.19 \mathrm{~m} / \mathrm{s}$ and friction path $S=342 \mathrm{~m}$. During the measurements, the depth of the worn-out groove on the projector screen was $12 \mathrm{~mm}$, or $12 / 77=0.15 \mathrm{~mm}$ taking in account the $\times 77$ magnification. The groove width was $2 a=205 / 77=2.66 \mathrm{~mm}$, respectively. The amount of wear calculated from formula (6) depending on the half-width of the groove, was $u_{W}=0.14 \mathrm{~mm}$. That is, discrepancy between the measured and calculated values did not exceed $7 \%$ which permits the use of the calculated dependence of the depth of the groove of a circular profile on the groove width

\section{The results of tests and calculations of wear of the sliding bearings}

To determine wear resistance parameters $C_{W}, n, p$ of the model (1) of the plain bearing wear, conical specimens of bronze $\mathrm{CuSn} 10 \mathrm{P}$ were tested. For statistical representativeness, each test cycle was carried out for 3 identical specimens. The averaged values obtained in measuring width of the worn-out groove are shown in Table 1 . The value of the coefficient of variation characterizing scatter of the obtained measurement results was in the range $v=0.15 \ldots 0.2$ which indicates sufficient homogeneity of the experimental sample.

Table 1: Results of measuring width of the wear groove and calculating the wear depth

\begin{tabular}{|c|c|c|c|c|}
\multirow{2}{*}{$S, \mathrm{~m}$} & \multicolumn{2}{|c|}{$V_{1}=0.19 \mathrm{~m} / \mathrm{s}$} & \multicolumn{2}{c|}{$V_{2}=0.38 \mathrm{~m} / \mathrm{s}$} \\
\cline { 2 - 5 } & $a, \mathrm{~mm}$ & $u_{W}, \mathrm{~mm}$ & $a, \mathrm{~mm}$ & $u_{W}, \mathrm{~mm}$ \\
\hline 115 & 0.4 & 0.01 & 0.45 & 0.012 \\
\hline 231 & 0.45 & 0.012 & 0.475 & 0.016 \\
\hline 346 & 0.465 & 0.014 & 0.55 & 0.018 \\
\hline 462 & 0.55 & 0.016 & 0.6 & 0.026 \\
\hline 577 & 0.55 & 0.019 & 0.625 & 0.031 \\
\hline 693 & 0.6 & 0.021 & 0.675 & 0.036 \\
\hline
\end{tabular}

Graphical interpretation of the test results, that is, the dependence of the half-width of the wear spot and their power approximation are shown in Fig. 5.

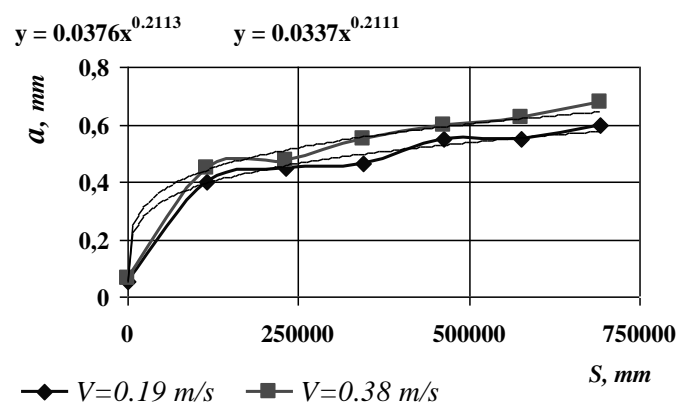

Fig. 5: Dependences of the half-width of the wear spot on the friction path and their power approximations

Fig. 5 also shows the power approximation with parameters and the magnitude of veracity of approximation $R$. Thus, to determine 
the parameter of influence of the slip velocity on the wear resistance $p$, the following parameters of approximating functions of the form (24): were obtained: $c_{1}=0.0337 ; \quad c_{2}=0.0376$; $\beta_{1} \approx \beta_{2}=\beta=0.21$.

Further, calculate the wear resistance parameters $C_{W}, n, p$ using formulas (23), (27), (28). As a result of calculation when testing steel sliding on bronze, parameters of wear resistance were obtained: $n=1.38 ; p=0.68 ; \mathrm{CW}_{\mathrm{W}}=9.625 \cdot 10^{-9}$. To obtain values of the wear resistance parameters as close as possible to the real operating conditions, it is necessary to conduct laboratory tests according to the proposed scheme under specified conditions.

Calculation will be carried out for materials of a friction pair "steel - bronze". Radial load on the bearing $Q=500 \mathrm{~N}$; hardness of bronze CuSn10P $H B=90 \mathrm{MPa}$; width and radius of the bearing: $b=20 \mathrm{~mm}, R=20 \mathrm{~mm}$; radial clearance $\Delta=0.1 \mathrm{~mm}$. Lubricant Magnum $15 \mathrm{~W}-40$ oil (TNK, Ukraine) was used with viscosity $v=40$ $\mathrm{mm}^{2} / \mathrm{s}$; coefficient of friction $f=0.08$. Sliding velocity was assumed equal to $1 \mathrm{~m} / \mathrm{s}$ which corresponds to the rotation frequency of the bearing shaft $475 \mathrm{~min}^{-1}$. The initial data were substituted into the formula for calculating wear (12) and the results of calculating wear as a function of the friction path are presented in Table 2 .

Table 2: Results of calculating wear $\mathrm{uW}$ of the sliding bearing as a function of friction path $\mathrm{S}$ and working time $\mathrm{t}$.

\begin{tabular}{|l|c|c|c|c|}
\hline $\mathrm{S}, \mathrm{km}$ & 10 & $10^{2}$ & $10^{3}$ & $10^{4}$ \\
\hline$t, \mathrm{hr}$ & 1.5 & 15 & 150 & 1500 \\
\hline$u_{W}, \mathrm{~mm}$ & 4 & 17 & 76 & 338 \\
\hline
\end{tabular}

Beside calculation of wear for the specified lifetime, dependence (12) allows one to analyze effect of design and operation parameters of bearings on their wear. This makes it possible to select optimal parameters of bearings at the stage of design preparation of the machine by the criterion of maximum wear resistance. Fig. 6 shows the graphs of influence of sliding velocity and load on the bearing wear obtained from the dependence (12).

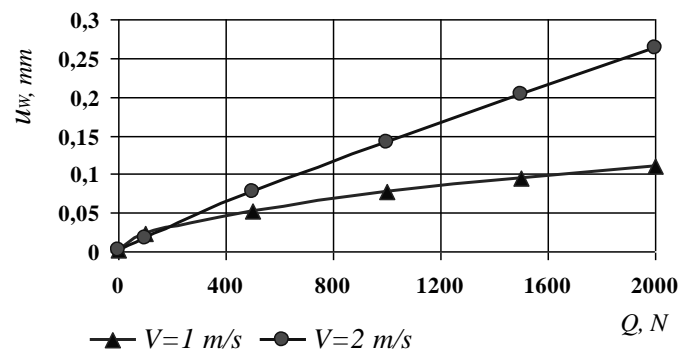

Fig. 6.: Effect of sliding velocity and load on wear of the slide bearing

Analysis of the obtained results has shown that all obtained dependences for the bearing wear were nonlinear.

\section{Discussion of the results obtained in model- ing of wear}

The resulting calculation model (12) for estimating wear of sliding bearings assumes a simple algorithm for obtaining wear values from the friction path under given operation conditions. This was achieved through the use of certain assumptions in solution of the wear-contact problem. Firstly, a uniform distribution of contact pressures over the wear spot was taken for average values. Secondly, difficult for integral-differential transformations trigonometric dependence of wear on the contact angle (3) was approximated by plain power dependence (5). As a rule, known solutions require numerical algorithms to overcome mathematical difficulties. At the same time, in solving the problem, this approach has allowed us to use a nonlinear model of wear from the determining factors (1) which adequately describes the actual wear processes. It is impossible to obtain numerical results using formula (12) without the values of the wear parameters $C_{W}, n, p$. That is, the wear model (12) can be applied in practice if an algorithm for determining parameters of this regularity is known. It is the parameters that enable quantitative estimation of the effect of determining factors (pressure and slip velocity). The wear parameters can only be established by the test results which makes the model approximated to the real tribosystem operation conditions.

The procedure for determining parameters of wear models was developed based on solution of an inverse wear-contact problem. In this case, dependences for calculating parameters (23), (27), (28) were obtained according to the adopted mathematical form of the wear law (1), the equilibrium condition (16), the geometric condition of continuity in the contact (17) and the wear test results. In this process, a proper choice of scheme of laboratory wear tests is very important. The scheme to be chosen for the tests should be one which mostly corresponds to the real tribounit as to its geometric and technological features. For example, the widely used four-ball test scheme is suitable for the tribounits in which a linear or dot contact takes place (toothed drives, cam mechanisms).

\section{Conclusions}

A model of wear of a sliding bearing in conditions of boundary friction was proposed in a form of a nonlinear dependence of the wear rate on the determining factors, i.e. contact pressure and sliding velocity. The wear model was presented in a form of dimensionless complexes taking into account geometric, kinematic, friction and lubrication characteristics of sliding bearings.

The wear-contact problem for cylindrical sliding bearings was solved. The dependence for average pressures in the bearing and the approximating function of linear wear on the contact arc were used as determining equations.

A calculation-experimental procedure for identifying parameters of wear resistance for the wear tests by the "cone - three balls" scheme was developed based on the two-factor model of wear.

\section{References}

[1] Chernets, M. V. (2015). Prediction of the life of a sliding bearing based on a cumulative wear model taking into account the lobing of the shaft contour. Journal of Friction and Wear, 36(2), 163-169. doi:10.3103/S1068366615020038

[2] Soldatenkov, I.A. (2010). Evolution of contact pressure during wear of the coating in a thrust sliding bearing. Journal of Friction and Wear, 31(2), 102-106. doi:10.3103/S1068366610020029

[3] Mezrin, A.M. (2009). Determining local wear equation based on friction and wear testing using a pin-on-disk scheme. Journal of Friction and Wear, 30(4), 242-245. doi:10.3103/S1068366609040035

[4] Dykha, A.V., Kuzmenko, A.G. (2016). Distribution of friction tangential stresses in the Courtney-Pratt experiment under Bowden's theory. Journal of Friction and Wear, 37 (4), 315-319. doi $10.3103 / \mathrm{s} 1068366616040061$

[5] Bulgarevich, S.B., Boiko, M.V., Lebedinskii, K.S., Marchenko D.Yu. (2014). Kinetics of sample wear on four-ball friction-testing machine using lubricants of different consistencies. Journal of Friction and Wear, 35(6), 531-537. doi:10.3103/S106836661406004X

[6] Dykha, A.V., Kuzmenko, A.G. (2015). Solution to the problem of contact wear for four-ball wear-testing scheme. Journal of Friction and Wear, 36(2), 138-143. doi: 10.3103/S1068366615020051

[7] Rezaei, A., Paepegem, W.V., Baets, P.D., Ost, W., Degrieck, J. (2012). Adaptive finite element simulation of wear evolution in radial sliding bearings. Wear, 296(1-2), 660-671. https://doi.org/10.1016/j.wear.2012.08.013

[8] Dykha, A. Determining the characteristics of viscous friction in the sliding supports using the method of pendulum [Text] / A. Dykha, V. Aulin, O. Makovkin, S. Posonskiy // Eastern-European Journal of Enterprise Technologies. - 2017. - Vol. 3, Issue 7 (87). - P. 4 10. doi: 10.15587/1729-4061.2017.99823

[9] Vynar, V.A., Dykha, M.O. (2013). Influence of the stress-strain state on the wear resistance of the surface of $40 \mathrm{Kh}$ Steel after discrete electromechanical treatment. Materials Science, 49 (3), 375 381. https://doi.org/10.1007/s11003-013-9625-Z 